\title{
Effects of Various Pre-Treatment and Cooking on the Levels of Biogenic Amines in Korean and Norwegian Mackerel
}

\author{
Yang-Su Kim ${ }^{1}$, Yuri Kim ${ }^{1,2}$, Hyunbeen Park ${ }^{1}$, Jooyeon Park ${ }^{1}$ and Kwang-Geun Lee ${ }^{1, *(D)}$ \\ 1 Department of Food Science and Biotechnology, Dongguk University-Seoul, 32, Dongguk-ro, Ilsandong-gu, \\ Goyang-si 10326, Gyeonggi-do, Korea; high_code@naver.com (Y.-S.K.); yuri@kfri.re.kr (Y.K.); \\ hbeen14@naver.com (H.P.); pjy041100@naver.com (J.P.) \\ 2 Food Certification Support Centre, Korea Food Research Institute, 245, Nongsaengmyeong-ro, Iseo-myeon, \\ Wanju-gun 55365, Jeollabuk-do, Korea \\ * Correspondence: kwglee@dongguk.edu; Tel.: +82-31-961-5142
}

Citation: Kim, Y.-S.; Kim, Y.; Park, H.; Park, J.; Lee, K.-G. Effects of Various Pre-Treatment and Cooking on the Levels of Biogenic Amines in Korean and Norwegian Mackerel. Foods 2021, 10, 2190. https://doi.org/10.3390/ foods10092190

Academic Editors: Maria Schirone and Pierina Visciano

Received: 12 August 2021

Accepted: 13 September 2021

Published: 15 September 2021

Publisher's Note: MDPI stays neutral with regard to jurisdictional claims in published maps and institutional affiliations.

Copyright: (c) 2021 by the authors. Licensee MDPI, Basel, Switzerland. This article is an open access article distributed under the terms and conditions of the Creative Commons Attribution (CC BY) license (https:// creativecommons.org/licenses/by/ $4.0 /)$.

\begin{abstract}
This study analyses the biogenic amines (BAs) formed in mackerel cooked by various methods and conditions. Five BAs, including tryptamine, $\beta$-phenylethylamine, putrescine, histamine, and spermidine, were analysed by high-performance liquid chromatography with UV detection. The level of total BAs was higher in the mackerel fillet $(108.14 \mu \mathrm{g} / \mathrm{g})$ than the headed and gutted fish $(91.58 \mu \mathrm{g} / \mathrm{g})$. Roasted, fried, and stewed mackerel recorded total BA concentrations of $54.28,82.25$, and $163.05 \mu \mathrm{g} / \mathrm{g}$, respectively. Stewed mackerel contained about 3-fold more BAs than roasted mackerel. The level of total BAs in mackerel increased significantly up to $190 \%$, $236 \%$ and $152 \%$ as the roasting temperature increased, upon frying, and as stewing temperature increased, respectively $(p<0.05)$.
\end{abstract}

Keywords: mackerel; biogenic amine; high-performance liquid chromatography; pre-treatment; cooking

\section{Introduction}

Mackerel is one of the most popular fish consumed in Korea. Safety issues for fishery products are increasing every year. Safety investigations on radioactive, heavy metal, and pesticide residues have been conducted for fishery products [1,2]. However, no study has yet conducted a detailed determination of biogenic amines (BAs) in cooked foods such as, for example, school meals. BAs are nitrogenous, low-molecular-weight organic bases with aromatic or heterocyclic structures. BAs are natural contaminants derived from the enzymatic decarboxylation of the amino acids concerned [3,4]. BAs can be formed in a variety of foods, such as cheese, sausage, fishery products, soybean products, and other fermented foods [5].

The BAs that affect the human body are tryptamine (TRP), histamine (HIS), putrescine (PUT), spermidine (SPD), and $\beta$-phenylethylamine (PHE), which are derived from amino acid precursors [5]. High levels of BAs can damage the human nervous and cardiovascular systems. BAs are known as potential precursors to carcinogenic nitrosamines [6-8]. Scombroid fish are characterised by the presence of histidine, a precursor of HIS in muscle tissue, more than other fish. Consuming food containing large amounts of HIS causes HIS poisoning, also called scombroid poisoning [9,10].

BAs are generally known to be heat-stable compounds. However, this is still controversial. Recent studies have indicated that the corresponding amino acid precursors can be formed by oxidative decarboxylation during the heating process [11]. Studies have reported reduced or increased BAs depending on the heat treatment temperature and time $[12,13]$.

There are several studies investigating concentrations of biogenic amines in raw mackerel [14-17]. Recent studies reported that concentrations of biogenic amines in Indian mackerel of the tropical region during storage at ambient $\left(25-29^{\circ} \mathrm{C}\right)$ and ice temperature 
$\left(0{ }^{\circ} \mathrm{C}\right)$ are related with changes of amino acids content and amines forming bacteria [18]. Although most people consume cooked mackerel, the contents of BAs from cooked mackerel have not been fully explored. Therefore, this study would be meaningful by analyzing the biogenic amine content of mackerel cooked in three different ways (roasting, frying, stewing).

In this study, to assess how various conditions can affect BAs in chub mackerel (Scomber japonicus), five BAs, including TRP, PHE, PUT, HIS, and SPD, formed in mackerel were analyzed considering its origin (Korea and Norway), pre-treatment (headed and gutted [H/G], fillet) and cooking method (roasted, fried, stewed).

\section{Materials and Methods}

\subsection{Chemicals and Materials}

Mackerel (S. japonicus from Korea and Norway) was purchased from Namyang Seafood Co. (Sungnam, Korea). Mackerel was purchased within a year of being caught and was kept at $-18^{\circ} \mathrm{C}$ until sample preparation. Canned tuna (Dongwon Industries Co., Seoul, Korea) was purchased in the general market in Seoul, Korea, and used to validate the five BAs.

Putrescine dihydrochloride, histamine dihydrochloride, TRP, 2-phenylethylamine, spermidine tri-hydrochloride, 1,7-diaminoheptane, perchloric acid, sodium hydrogen carbonate, and ammonium hydroxide were purchased from Sigma Aldrich Chemical Co. (St. Louis, MO, USA). HPLC-grade water and acetonitrile were purchased from J.T. Baker (Phillips, NJ, USA). The dansyl chloride reagent used for derivatisation was purchased from Tokyo Chemical Industry Co., Ltd. (Tokyo, Japan).

\subsection{Validation of the Analysis of BAs}

Canned tuna was used to validate the five BAs since it has a similar fatty acid composition to mackerel and has not been reported to have BAs. For method validation, linearity (coefficient of determination, $\mathrm{R}^{2}$ of the calibration curve), limit of detection (LOD), limit of quantitation (LOQ), recovery (\%), and precision (relative standard deviation, RSD \%) were determined. The calibration curve of the five BAs in canned tuna was prepared by measuring seven concentrations of the BAs standard solution $(0,1,3,5,10,50,100$, $300 \mu \mathrm{g} / \mathrm{g})$. LOD and LOQ were calculated by the respective equations, $3.14 \times$ standard deviation $(\sigma 1)$ /slope factor and $10 \times$ standard deviation $(\sigma 1) /$ slope factor of the BAs calibration curve derived from eight points $(0,1,3,5,10,50,100,300 \mu \mathrm{g} / \mathrm{g})$. For accuracy $(\%)$ and precision, the level of BAs were determined in canned tuna samples without internal standards. The intra-day accuracy and precision were analysed on one day by performing five replicates at each level of the BAs $(10,50$, and $100 \mu \mathrm{g} / \mathrm{g})$. The inter-day accuracy and precision were tested once a day for 5 days at each level of the BAs $(10,50$, and $100 \mu \mathrm{g} / \mathrm{g})$.

\subsection{Preparation of Mackerel Samples}

A total of 40 samples of mackerel, as shown in Table 1, were prepared for BAs analysis by origin (Korea and Norway), pre-treatment (H/G, fillet), cooking method (roasted, fried, stewed), temperature, and time conditions. Each treatment has 3 replications, and the total analytical samples were 120 . 
Table 1. Preparation of 40 mackerel samples.

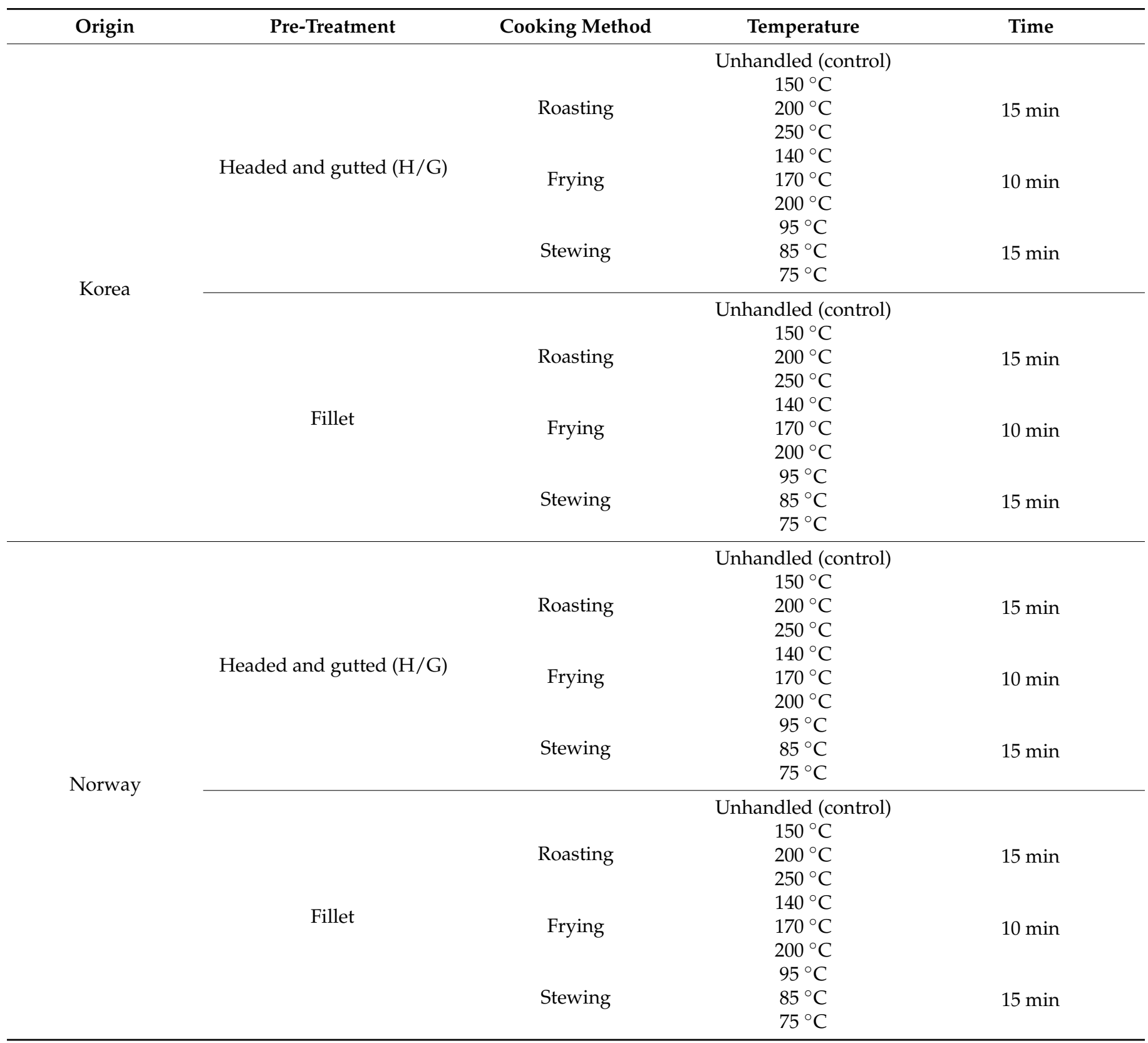

Frozen whole mackerel was thawed in water for $30 \mathrm{~min}$ before the experiments. H/G mackerel has viscera (guts) and head removed. Mackerel fillets were cut along the entire side of the fish, removing the spine and most bones from the meat. The cooking method applied to mackerel was conducted with reference to the Korean Dietetic Association (The Korean Dietary Association, 2007). Roasted mackerel was prepared by roasting $50 \mathrm{~g}$ of mackerel at 150, 200, and $250{ }^{\circ} \mathrm{C}$ for $15 \mathrm{~min}$ in an oven (HSB-N361B, Samsung, Seoul, Korea). For fried mackerel, $50 \mathrm{~g}$ of mackerel was fried in soybean oil (Sajo Haepyo, Seoul, Korea) at 140,170 , and $200{ }^{\circ} \mathrm{C}$ for $10 \mathrm{~min}$ using a fryer (DK-260, Delki, Seoul, Korea). For stewed mackerel, $50 \mathrm{~g}$ of mackerel, $200 \mathrm{~mL}$ of soy sauce (Sampyo, Seoul, Korea), and $200 \mathrm{~mL}$ of water were combined and boiled at 75,85 , and $95{ }^{\circ} \mathrm{C}$ for $15 \mathrm{~min}$. A calibrated infrared thermometer (SATO-8700, SATO, Seoul, Korea) was used for temperature measurement in all cooking procedures. Prepared samples were stored at $4{ }^{\circ} \mathrm{C}$ for $3 \mathrm{~h}$ until analysis. The sample preparation of mackerel is described in Table 1. 


\subsection{Sample Preparation for BAs Analysis}

\subsubsection{Preparation of Sample Extracts}

The BAs analysis of mackerel was carried out according to the procedure proposed by Lee et al. [19]. In a $50 \mathrm{~mL}$ conical tube, $5 \mathrm{~mL}$ of mackerel sample was added to $20 \mathrm{~mL}$ of $0.4 \mathrm{M}$ perchloric acid, and $50 \mu \mathrm{L}$ of 1,7-diaminoheptane $(10 \mathrm{~g} / \mathrm{L})$ as the internal standard. This mixture was homogenised using a vortex mixer, then left to react in a cold chamber for $2 \mathrm{~h}$, followed by centrifugation at $12,000 \mathrm{rpm}, 4{ }^{\circ} \mathrm{C}$, for $10 \mathrm{~min}$. The supernatant was collected, and the residue was re-extracted with $0.4 \mathrm{M}$ perchloric acid of the same volume as the previous experimental method (second extraction). After pooling the two supernatants, the final volume was adjusted to $50 \mathrm{~mL}$ with $0.4 \mathrm{M}$ perchloric acid and filtered through Whatman filter paper No. 1 (Whatman Ltd., Little Chalfont, UK) to progress the derivatisation.

\subsubsection{Derivatisation of Extracted Sample}

BAs were derivatised according to the previously published method [20]. One millilitre of the extracted sample was mixed with $200 \mu \mathrm{L}$ of $2 \mathrm{M} \mathrm{NaOH}$ and $300 \mu \mathrm{L}$ of saturated sodium bicarbonate. For derivatisation, $2 \mathrm{~mL}$ of dansyl chloride solution was added to the mixture, followed by incubation of the mixture at $40{ }^{\circ} \mathrm{C}$ for $45 \mathrm{~min}$. Ammonium hydroxide $(25 \%, 100 \mu \mathrm{L})$ was added to stop the reaction and remove the residual dansyl chloride. After that, the mixture was stored in the dark at room temperature for $30 \mathrm{~min}$, and then acetonitrile was added to adjust the final volume to $5 \mathrm{~mL}$. The mixture was centrifuged at $3500 \mathrm{rpm}$ for $5 \mathrm{~min}$. The resultant supernatant was filtered through a syringe filter $(25 \mathrm{~mm}$, $0.2-\mu \mathrm{m}$ pore size; Whatman Ltd.) and stored at $-25{ }^{\circ} \mathrm{C}$ until analysis by HPLC-UV.

\subsection{Analysis of BAs in Mackerel by HPLC-UV}

The BAs were quantitated using an HPLC 1200 Series (Agilent Technologies, Santa Clara, CA, USA) equipped with a UV-Vis detector and Nova-Pack C18 column $(3.9 \times 150 \mathrm{~mm}, 4 \mu \mathrm{m})$. The mobile phases were $0.1 \mathrm{M}$ ammonium acetate (solvent $\mathrm{A}$ ) and acetonitrile (solvent $\mathrm{B}$ ) at the flow rate of $1 \mathrm{~mL} / \mathrm{min}$ with gradient elution for $25 \mathrm{~min}$. The injection volume was $20 \mu \mathrm{L}$. The sample was detected by UV at a wavelength of $254 \mathrm{~nm}$. After $25 \mathrm{~min}$, the gradient was re-adjusted to $50 \%$ solvent $\mathrm{A}$ and $50 \%$ solvent $\mathrm{B}$.

\subsection{Statistical Analysis}

The results were expressed as mean \pm standard deviation of three measurements. Triplicate analyses were performed for each mackerel sample. Data were evaluated for origin, cooking methods, and temperature by one-way analysis of variance (ANOVA) and Duncan's multiple range test using IBM SPSS Statistics 23 (IBM Co., Armonk, NY, USA). We ran a One-way ANOVA followed by Duncan's multiple range test because we were interested in the effect of each independent variable (origin, cooking method, temperature) rather than the interaction between them. While it seems like temperature is nested in the cooking method, each variable was specifically selected for each cooking method; therefore, cooking method and temperature need to be treated independently.

\section{Results and Discussion}

\subsection{Validation of Analytical Method for BAs}

For method validation, the results of linearity (coefficient of determination, $\mathrm{R}^{2}$ of the calibration curve), limit of detection (LOD), limit of quantitation (LOQ), recovery (\%), and precision (relative standard deviation, RSD \%) can be found in Table 2. Suitable linearity for BAs was observed at all concentrations. $\left(\mathrm{R}^{2}>0.99\right)$ The measured LOD and LOQ ranges of the five BAs were $0.78-1.45$ and $1.65-4.40 \mu \mathrm{g} / \mathrm{g}$, respectively. Recovery (\%) and precision (\%) were measured, respectively, using three concentrations $(10,50,100 \mu \mathrm{g} / \mathrm{g})$ of the BAs standard solutions. The recovery rate ranged from $85.78 \%$ to $118.06 \%$, the inter-day and intra-day precision values ranged from $0.95 \%$ to $9.68 \%$ and from $0.82 \%$ to $8.65 \%$, respectively. The validation data of this study was similar to the previous reports $[7,19]$. 
Table 2. Validation for the analysis of BAs (calibration curve, linearity, LOD, LOQ, RSD (\%), recovery rate).

\begin{tabular}{|c|c|c|c|c|c|c|c|c|c|c|c|c|c|}
\hline \multirow{4}{*}{ BAs ${ }^{b}$} & \multirow{2}{*}{\multicolumn{2}{|c|}{ Calibration Curve $^{a}$}} & \multirow{4}{*}{$\begin{array}{l}\text { LOD }^{c} \\
(\mu g / g)\end{array}$} & \multirow{4}{*}{$\begin{array}{c}\operatorname{LOQ}^{d} \\
(\mu g / g)\end{array}$} & \multicolumn{6}{|c|}{ Precision (RSD \%) } & \multirow{2}{*}{\multicolumn{3}{|c|}{ Recovery Rate (\%) }} \\
\hline & & & & & & ter-Da & & & tra-Da & & & & \\
\hline & \multirow{2}{*}{$\begin{array}{l}\text { Equation } \\
(y=a x+b)\end{array}$} & \multirow{2}{*}{$\begin{array}{l}\text { Linearity } \\
\quad\left(\mathbf{R}^{2}\right)\end{array}$} & & & \multicolumn{3}{|c|}{ Concentration $(\mu \mathrm{g} / \mathrm{g})$} & \multicolumn{3}{|c|}{ Concentration $(\mu \mathrm{g} / \mathrm{g})$} & \multicolumn{3}{|c|}{ Concentration $(\mu \mathrm{g} / \mathrm{g})$} \\
\hline & & & & & 10 & 50 & 100 & 10 & 50 & 100 & 10 & 50 & 100 \\
\hline TRP & $y=0.0123 x+0.0493$ & 0.9996 & 0.78 & 2.49 & 3.46 & 7.64 & 4.66 & 8.65 & 6.34 & 3.53 & 98.23 & 106.38 & 89.62 \\
\hline PHE & $y=0.0113 x+0.0464$ & 0.9997 & 0.52 & 1.65 & 9.68 & 3.73 & 0.95 & 1.54 & 4.81 & 2.86 & 85.78 & 107.62 & 95.87 \\
\hline PUT & $y=0.0046 x+0.088$ & 0.9985 & 0.84 & 2.69 & 4.89 & 2.26 & 1.84 & 2.76 & 0.82 & 2.94 & 113.25 & 118.06 & 102.85 \\
\hline HIS & $y=0.0039 x+0.0165$ & 0.9999 & 0.65 & 2.08 & 2.74 & 1.08 & 2.62 & 1.88 & 3.46 & 4.85 & 94.85 & 97.68 & 93.85 \\
\hline SPD & $y=0.0009 x+0.0065$ & 0.9991 & 1.45 & 4.4 & 5.85 & 1.87 & 3.87 & 6.74 & 3.08 & 5.64 & 103.51 & 102.68 & 111.93 \\
\hline
\end{tabular}

${ }^{a}$ Range of calibration curve was measured at seven points: $0.1,3.5,10,50,100,300(\mu \mathrm{g} / \mathrm{g}){ }^{\mathrm{b}}$ Trp: tryptamine, Phe: $\beta$-phenylethylamine, Put: putrescine,

His: histamine, Spd: spermidine. ${ }^{\mathrm{c}} \mathrm{LOD}=3.14 \times$ Standard deviation/Slope $(\mu \mathrm{g} / \mathrm{g}) .{ }^{\mathrm{d}} \mathrm{LOQ}=10 \times$ Standard deviation/Slope $(\mu \mathrm{g} / \mathrm{g})$.

\subsection{Analysis of BAs Level in Mackerel}

The concentrations of the five BAs detected in the 40 mackerel samples are presented in Tables 3-5. Though the stored frozen time of the fish before the experiment could have affected the experiment, the effect is not expected to confound the results since it applies to both origins and across treatments. Based on the cooking method, the concentration of the total BAs was highest in stewed $(163.05 \mu \mathrm{g} / \mathrm{g})$, followed by fried $(82.25 \mu \mathrm{g} / \mathrm{g})$ and roasted $(54.28 \mu \mathrm{g} / \mathrm{g})$. The highest concentration of total BAs $(192.53 \mu \mathrm{g} / \mathrm{g})$ was measured in the fillet of mackerel from Norway, stewed at $95{ }^{\circ} \mathrm{C}$. The lowest total concentration $(33.09 \mu \mathrm{g} / \mathrm{g})$ was measured in the $\mathrm{H} / \mathrm{G}$ fish from Korea, fried at $140{ }^{\circ} \mathrm{C}$.

Table 3. Concentrations of BAs in roasted mackerel samples.

\begin{tabular}{|c|c|c|c|c|c|c|c|c|}
\hline Origin & $\begin{array}{l}\text { Pretreatment \& Cooking } \\
\text { Method }\end{array}$ & Temperature & $\begin{array}{l}\text { Tryptamine } \\
\text { (TRP) }\end{array}$ & $\begin{array}{c}\beta \text {-Phenyl } \\
\text { Ethylamine (PHE) }\end{array}$ & $\begin{array}{l}\text { Putrescine } \\
\text { (PUT) }\end{array}$ & $\begin{array}{l}\text { Histamine } \\
\text { (HIS) }\end{array}$ & $\begin{array}{l}\text { Spermidine } \\
\text { (SPD) }\end{array}$ & Total BAs \\
\hline \multirow{8}{*}{ Korea } & $\begin{array}{l}\text { Control(H/G) } \\
\text { Control(fillet) }\end{array}$ & & $8.03 \pm 0.95$ & $4.01 \pm 0.41$ & $22.32 \pm 2.1$ & $6.34 \pm 0.17$ & $2.30 \pm 0.22$ & $43.00 \pm 3.24$ \\
\hline & Control(fillet) & & $8.3 \pm 0.77$ & $5.27 \pm 0.32$ & $21.62 \pm 2.15$ & $8.38 \pm 0.05$ & $3.6 \pm 0.11$ & $47.18 \pm 1.63$ \\
\hline & \multirow{3}{*}{$\begin{array}{l}\text { Headed and gutted, } \\
\text { Roasted }\end{array}$} & $150^{\circ} \mathrm{C}$ & $5.10 \pm 0.09 \mathrm{a}, \mathrm{A}, \alpha$ & $7.07 \pm 0.25 \mathrm{~b}, \mathrm{~B}, \beta \gamma$ & $20.95 \pm 2.8 \mathrm{a}, \mathrm{B} \alpha$ & $6.45 \pm 0.26^{\mathrm{a}, \mathrm{A}, \alpha}$ & $2.44 \pm 0.13 \mathrm{ab}, \mathrm{A}, \alpha$ & $42.01 \pm 3.27$ \\
\hline & & $200{ }^{\circ} \mathrm{C}$ & $6.74 \pm 0.35 \mathrm{a}, \mathrm{A}, \beta \gamma$ & $6.53 \pm 0.24 \mathrm{a}, \mathrm{A}, \alpha$ & $26.23 \pm 3.32 \mathrm{a}, \mathrm{B}, \beta$ & $7.01 \pm 0.26 \mathrm{ab}, \mathrm{A}, \alpha \beta$ & $2.86 \pm 0.06 \mathrm{a}, \mathrm{A}, \alpha \beta$ & $49.37 \pm 4.06$ \\
\hline & & $250^{\circ} \mathrm{C}$ & $6.07 \pm 0.5 \mathrm{a}, \mathrm{A}, \mathrm{\beta}$ & $6.41 \pm 0.62 \mathrm{a}, \mathrm{A}, \alpha \beta$ & $30.40 \pm 0.91 \mathrm{~b}, \mathrm{AB}, \gamma$ & $7.49 \pm 0.16 \mathrm{ab}, \mathrm{A}, \beta \gamma$ & $3.31 \pm 0.13 \mathrm{a}, \mathrm{A}, \beta \gamma$ & $53.68 \pm 1.07$ \\
\hline & \multirow{3}{*}{ Fillet, Roasted } & $150^{\circ} \mathrm{C}$ & $5.20 \pm 0.39 \mathrm{a}, \mathrm{B}, \alpha$ & $5.95 \pm 0.7 \mathrm{a}, \mathrm{A}, \alpha$ & $11.43 \pm 0.6 \mathrm{a}, \mathrm{A}, \alpha$ & $9.04 \pm 0.65 \mathrm{~b}, \mathrm{~B}, \alpha$ & $2.98 \pm 0.61 \mathrm{~b}, \mathrm{~B}, \alpha$ & $34.60 \pm 1.65$ \\
\hline & & $200^{\circ} \mathrm{C}$ & $14.08 \pm 0.79 \mathrm{a}, \mathrm{B}, \alpha \beta$ & $6.86 \pm 0.39 \mathrm{~b}, \mathrm{~B}, \alpha \beta$ & $13.05 \pm 0.53 \mathrm{ab}, \mathrm{A}, \alpha \beta$ & $11.13 \pm 0.24 \mathrm{a}, \mathrm{B}, \alpha \beta$ & $3.77 \pm 0.34 \mathrm{~b}, \mathrm{~B}, \beta$ & $48.89 \pm 1.17$ \\
\hline & & $250^{\circ} \mathrm{C}$ & $22.29 \pm 1.57^{a}, \mathrm{~B}, \beta \gamma$ & $7.99 \pm 0.53 \mathrm{a}, \mathrm{B}, \beta \gamma$ & $14.91 \pm 0.76 \mathrm{a}, \mathrm{A}, \mathrm{\beta}$ & $13.84 \pm 1.06 \mathrm{ab}, \mathrm{B}, \gamma$ & $3.88 \pm 0.37 \mathrm{~b}, \mathrm{~B}, \beta \gamma$ & $62.91 \pm 2.35$ \\
\hline \multirow{8}{*}{ Norway } & Control(H/G) & & $6.46 \pm 0.6$ & $4.17 \pm 0.09$ & $28.08 \pm 3.23$ & $6.49 \pm 0.05$ & $5.61 \pm 0.5$ & $50.80 \pm 3.49$ \\
\hline & Control(fillet) & & $9.85 \pm 0.81$ & $5.66 \pm 0.41$ & $23.74 \pm 0.26$ & $8.82 \pm 0.07$ & $6.77 \pm 0.28$ & $54.83 \pm 0.94$ \\
\hline & \multirow{3}{*}{$\begin{array}{l}\text { Headed and gutted, } \\
\text { Roasted }\end{array}$} & $150^{\circ} \mathrm{C}$ & $5.96 \pm 0.5 \mathrm{~b}, \mathrm{~A}, \alpha$ & $5.36 \pm 0.1 \mathrm{a}, \mathrm{A}, \alpha$ & $22.10 \pm 1.24 \mathrm{ab}, \mathrm{B}, \alpha$ & $6.35 \pm 0.23 \mathrm{a}, \mathrm{A}, \alpha$ & $2.28 \pm 0.06^{a}, \mathrm{AB}, \alpha$ & $42.06 \pm 1.8$ \\
\hline & & $200^{\circ} \mathrm{C}$ & $7.06 \pm 0.36^{a}, \mathrm{~A}, \alpha \beta$ & $8.56 \pm 0.38 \mathrm{~b}, \mathrm{~B}, \beta \gamma$ & $27.93 \pm 3.34 \mathrm{~b}, \mathrm{~B}, \beta$ & $6.66 \pm 0.53 \mathrm{a}, \mathrm{A}, \alpha$ & $4.04 \pm 0.14 \mathrm{~b}, \mathrm{~B}, \beta \gamma$ & $54.25 \pm 2.55$ \\
\hline & & $250^{\circ} \mathrm{C}$ & $7.35 \pm 0.84 \mathrm{~b}, \mathrm{~A}, \alpha \beta$ & $7.62 \pm 1.07 \mathrm{~b}, \mathrm{~A}, \beta$ & $29.10 \pm 0.57 \mathrm{a}, \mathrm{b}, \gamma$ & $7.06 \pm 0.07 \mathrm{a}, \mathrm{A}, \alpha \beta$ & $4.35 \pm 0.34 \mathrm{~b}, \mathrm{~B}, \gamma$ & $55.48 \pm 2.27$ \\
\hline & \multirow{3}{*}{ Fillet, Roasted } & $150^{\circ} \mathrm{C}$ & $18.28 \pm 1.41 \mathrm{~b}, \mathrm{~B}, \alpha$ & $7.19 \pm 0.3 \mathrm{~b}, \mathrm{~B}, \alpha$ & $13.24 \pm 0.29 \mathrm{ab}, \mathrm{A}, \alpha \beta$ & $8.32 \pm 0.68^{a, B, \alpha}$ & $2.13 \pm 0.07 \mathrm{a}, \mathrm{A}, \alpha$ & $49.17 \pm 0.66$ \\
\hline & & $200{ }^{\circ} \mathrm{C}$ & $28.10 \pm 2.99 \mathrm{~b}, \mathrm{~B}, \beta$ & $7.80 \pm 0.25 \mathrm{a}, \mathrm{A}, \alpha \beta$ & $15.53 \pm 0.88 \mathrm{~b}, \mathrm{~A}, \mathrm{\beta}$ & $12.04 \pm 0.73 \mathrm{~b}, \mathrm{~B}, \mathrm{\beta}$ & $2.15 \pm 0.07 \mathrm{a}, \mathrm{A}, \alpha$ & $65.62 \pm 3.44$ \\
\hline & & $250{ }^{\circ} \mathrm{C}$ & $31.11 \pm 2.04 \mathrm{~b}, \mathrm{~B}, \beta$ & $10.16 \pm 0.75 \mathrm{~b}, \mathrm{~B}, \gamma$ & $34.08 \pm 2.68 \mathrm{~b}, \mathrm{~B}, \gamma$ & $14.42 \pm 0.91 \mathrm{~b}, \mathrm{~B}, \gamma$ & $3.55 \pm 0.15 \mathrm{a}, \mathrm{A}, \mathrm{B}$ & $93.32 \pm 1.56$ \\
\hline
\end{tabular}

Mean Values with three types of superscripts: ${ }^{a, b}$ _indicate significant differences in origin; ${ }^{A}, \mathrm{~B}$ _-indicate significant differences in pre-treatment; ${ }^{\alpha, \beta, \gamma}$ - indicate significant differences in Temperature. The significant differences according to Duncan's test $(p<0.05)$.

Table 4. Concentrations of BAs in fried mackerel samples.

\begin{tabular}{|c|c|c|c|c|c|c|c|c|}
\hline Origin & $\begin{array}{l}\text { Pretreatment \& Cooking } \\
\text { Method }\end{array}$ & Temperature & $\begin{array}{l}\text { Tryptamine } \\
\text { (TRP) }\end{array}$ & $\begin{array}{c}\beta \text {-Phenyl } \\
\text { Ethylamine (PHE) }\end{array}$ & $\begin{array}{l}\text { Putrescine } \\
\text { (PUT) }\end{array}$ & $\begin{array}{l}\text { Histamine } \\
\text { (HIS) }\end{array}$ & $\begin{array}{l}\text { Spermidine } \\
\text { (SPD) }\end{array}$ & Total BAs \\
\hline \multirow{8}{*}{ Korea } & Control(H/G) & & $8.03 \pm 0.95$ & $4.01 \pm 0.41$ & $22.32 \pm 2.1$ & $6.34 \pm 0.17$ & $2.30 \pm 0.22$ & $43.00 \pm 3.24$ \\
\hline & Control(fillet) & & $8.3 \pm 0.77$ & $5.27 \pm 0.32$ & $21.62 \pm 2.15$ & $8.38 \pm 0.05$ & $3.6 \pm 0.11$ & $47.18 \pm 1.63$ \\
\hline & \multirow{3}{*}{ Headed and gutted, Fried } & $140^{\circ} \mathrm{C}$ & $4.12 \pm 0.42 \mathrm{a}, \mathrm{A}, \alpha$ & $5.92 \pm 0.29 \mathrm{a}, \mathrm{A}, \alpha$ & $12.22 \pm 0.91 \mathrm{a}, \mathrm{A}, \alpha$ & $8.32 \pm 0.95$ & $2.51 \pm 0.53 \mathrm{a}, \mathrm{A}, \alpha$ & $33.09 \pm 2.84$ \\
\hline & & $170^{\circ} \mathrm{C}$ & $12.88 \pm 0.48 \mathrm{a}, \mathrm{A}, \mathrm{B}$ & $11.08 \pm 0.75 \mathrm{~b}, \mathrm{~A}, \mathrm{\beta}$ & $16.45 \pm 1.92 \mathrm{a}, \mathrm{A}, \mathrm{\beta}$ & $10.78 \pm 0.69 \mathrm{a}, \mathrm{A}, \beta$ & $3.71 \pm 0.3 \mathrm{a}, \mathrm{A}, \beta$ & $54.89 \pm 3.49$ \\
\hline & & $200^{\circ} \mathrm{C}$ & $17.70 \pm 2.3 \mathrm{a}, \mathrm{A}, \gamma$ & $20.83 \pm 0.69 \mathrm{a}, \mathrm{A}, \gamma$ & $20.00 \pm 2.27 \mathrm{~b}, \mathrm{~A}, \gamma$ & $13.86 \pm 0.88 \mathrm{a}, \mathrm{A}, \gamma$ & $4.90 \pm 0.62 \mathrm{a}, \mathrm{A}, \gamma$ & $77.28 \pm 5.35$ \\
\hline & \multirow{3}{*}{ Fillet, Fried } & $140^{\circ} \mathrm{C}$ & $31.30 \pm 3.27 \mathrm{~b}, \mathrm{~B}, \beta$ & $19.20 \pm 2.28 \mathrm{~b}, \mathrm{~B}, \beta$ & $34.02 \pm 4.25 \mathrm{~b}, \mathrm{~B}, \alpha$ & $15.16 \pm 1.53^{\mathrm{a}, \mathrm{B}, \alpha}$ & $10.09 \pm 0.77 \mathrm{~b}, \mathrm{~B}, \gamma$ & $109.77 \pm 2.43$ \\
\hline & & $170^{\circ} \mathrm{C}$ & $29.96 \pm 1.99 \mathrm{~b}, \mathrm{~B}, \alpha$ & $16.96 \pm 0.74 \mathrm{a}^{\mathrm{a}, \mathrm{B}, \alpha}$ & $22.91 \pm 1.14 \mathrm{~b}, \mathrm{~B}, \beta$ & $22.70 \pm 0.16 \mathrm{a}, \mathrm{A}, \alpha$ & $9.30 \pm 0.69 \mathrm{~b}, \mathrm{~B}, \beta$ & $101.84 \pm 3.93$ \\
\hline & & $200^{\circ} \mathrm{C}$ & $45.51 \pm 5.59 \mathrm{a}, \mathrm{AB}, \gamma$ & $22.39 \pm 1.97 \mathrm{~b}, \mathrm{~B}, \gamma$ & $31.25 \pm 1.06^{\mathrm{b}, \mathrm{B}, \beta}$ & $40.20 \pm 3.8^{\mathrm{b}, \mathrm{B}, \beta}$ & $7.22 \pm 0.18 \mathrm{~b}, \mathrm{~B}, \alpha$ & $146.58 \pm 6.43$ \\
\hline \multirow{8}{*}{ Norway } & Control(H/G) & & $6.46 \pm 0.6$ & $4.17 \pm 0.09$ & $28.08 \pm 3.23$ & $6.49 \pm 0.05$ & $5.61 \pm 0.5$ & $50.80 \pm 3.49$ \\
\hline & Control(fillet) & & $9.85 \pm 0.81$ & $5.66 \pm 0.41$ & $23.74 \pm 0.26$ & $8.82 \pm 0.07$ & $6.77 \pm 0.28$ & $54.83 \pm 0.94$ \\
\hline & \multirow{3}{*}{ Headed and gutted, Fried } & $140^{\circ} \mathrm{C}$ & $9.33 \pm 0.4 \mathrm{~b}, \mathrm{~A}, \alpha$ & $7.79 \pm 0.77 \mathrm{a}, \mathrm{A}, \alpha$ & $13.49 \pm 1.26^{b, A}, \alpha$ & $8.54 \pm 0.37 \mathrm{~b}, \mathrm{~A}, \alpha$ & $2.87 \pm 0.27 \mathrm{~b}, \mathrm{~A}, \alpha$ & $42.03 \pm 1.33$ \\
\hline & & $170^{\circ} \mathrm{C}$ & $19.89 \pm 2.2^{b, A}, \beta$ & $10.21 \pm 0.79 \mathrm{a}, \mathrm{A}, \beta$ & $17.37 \pm 1.63 \mathrm{~b}, \mathrm{~A}, \beta$ & $11.52 \pm 0.75 \mathrm{a}, \mathrm{A}, \beta$ & $8.29 \pm 0.88 \mathrm{a}, \mathrm{B}, \beta$ & $67.28 \pm 5.55$ \\
\hline & & $200^{\circ} \mathrm{C}$ & $24.32 \pm 2.03 \mathrm{~b}, \mathrm{~A}, \gamma$ & $32.27 \pm 3.04 \mathrm{~b}, \mathrm{~B}, \gamma$ & $18.52 \pm 1.68 \mathrm{a}, \mathrm{A}, \gamma$ & $15.30 \pm 1.94 \mathrm{~b}, \mathrm{~A}, \gamma$ & $8.91 \pm 0.77^{\mathrm{b}, \mathrm{B}, \gamma}$ & $99.32 \pm 4.89$ \\
\hline & \multirow{3}{*}{ Fillet, Fried } & $140^{\circ} \mathrm{C}$ & $16.82 \pm 2.19 \mathrm{a}, \mathrm{B}, \alpha$ & $15.70 \pm 1.66 \mathrm{a}, \mathrm{B}, \beta$ & $20.48 \pm 1.08 \mathrm{a}, \mathrm{B}, \beta$ & $10.29 \pm 0.37 \mathrm{a}, \mathrm{B}, \alpha$ & $6.04 \pm 0.06^{\mathrm{a}, \mathrm{B}, \gamma}$ & $69.33 \pm 5.12$ \\
\hline & & $170^{\circ} \mathrm{C}$ & $24.19 \pm 3.03 \mathrm{a}, \mathrm{B}, \beta$ & $21.55 \pm 2.07 \mathrm{~b}, \mathrm{~B}, \gamma$ & $18.82 \pm 2.15 \mathrm{a}, \mathrm{B}, \alpha$ & $14.83 \pm 1.12 \mathrm{~b}, \mathrm{~B}, \beta$ & $5.93 \pm 0.22 \mathrm{~b}, \mathrm{~A}, \beta$ & $85.31 \pm 3.83$ \\
\hline & & $200{ }^{\circ} \mathrm{C}$ & $37.63 \pm 5.02 \mathrm{a}, \mathrm{B}, \gamma$ & $14.97 \pm 1.64 \mathrm{a}, \mathrm{A}, \alpha$ & $21.19 \pm 1.96^{\mathrm{a}, \mathrm{B}, \beta}$ & $24.32 \pm 2.05 \mathrm{a}, \mathrm{B}, \gamma$ & $2.16 \pm 0.11 \mathrm{a}, \mathrm{A}, \alpha$ & $100.28 \pm 7.4$ \\
\hline
\end{tabular}

Mean Values with three types of superscripts: ${ }^{a, b}$ —indicate significant differences in origin; ${ }^{A, B}$ _indicate significant differences in pre-treatment; $\alpha, \beta, \gamma$ —indicate significant differences in Temperature. The significant differences according to Duncan's test $(p<0.05)$. 
Table 5. Concentrations of BAs in stewed mackerel samples.

\begin{tabular}{|c|c|c|c|c|c|c|c|c|}
\hline Origin & $\begin{array}{l}\text { Pretreatment \& } \\
\text { Cooking Method }\end{array}$ & Temperature & $\begin{array}{l}\text { Tryptamine } \\
\text { (TRP) }\end{array}$ & $\begin{array}{c}\beta \text {-Phenyl } \\
\text { Ethylamine (PHE) }\end{array}$ & $\begin{array}{l}\text { Putrescine } \\
\text { (PUT) }\end{array}$ & $\begin{array}{c}\text { Histamine } \\
\text { (HIS) }\end{array}$ & $\begin{array}{l}\text { Spermidine } \\
\text { (SPD) }\end{array}$ & Total BAs \\
\hline \multirow{8}{*}{ Korea } & $\begin{array}{l}\text { Control(H/G) } \\
\text { Control(fillet) }\end{array}$ & & $\begin{array}{l}8.03 \pm 0.95 \\
8.3 \pm 0.77\end{array}$ & $4.01 \pm 0.41$ & $22.32 \pm 2.1$ & $6.34 \pm 0.17$ & $2.30 \pm 0.22$ & $43.00 \pm 3.24$ \\
\hline & Control(fillet) & & $8.3 \pm 0.77$ & $5.27 \pm 0.32$ & $21.62 \pm 2.15$ & $8.38 \pm 0.05$ & $3.6 \pm 0.11$ & $47.18 \pm 1.63$ \\
\hline & \multirow{3}{*}{$\begin{array}{l}\text { Headed and gutted, } \\
\text { Stewed }\end{array}$} & $75^{\circ} \mathrm{C}$ & $17.41 \pm 1.06 \mathrm{a}, \mathrm{A}, \alpha$ & $24.62 \pm 4.35 \mathrm{a}, \mathrm{AB}, \alpha$ & $43.11 \pm 4.29 \mathrm{~b}, \mathrm{~B}, \alpha$ & $58.18 \pm 6.14 \mathrm{~b}, \mathrm{~B}, \alpha$ & $8.39 \pm 0.83 \mathrm{~b}, \mathrm{~B}, \alpha \beta$ & $151.70 \pm 4.35$ \\
\hline & & $85^{\circ} \mathrm{C}$ & $22.21 \pm 1.39 \mathrm{~b}, \mathrm{~B}, \beta$ & $36.88 \pm 2.83 \mathrm{a}, \mathrm{A}, \alpha$ & $42.39 \pm 3.14^{\mathrm{a}, \mathrm{A}, \mathrm{\beta}}$ & $64.81 \pm 2.28 \mathrm{a}, \mathrm{A}, \alpha \beta$ & $8.66 \pm 0.21 \mathrm{~b}, \mathrm{AB}, \beta \gamma$ & $174.94 \pm 9.18$ \\
\hline & & $95^{\circ} \mathrm{C}$ & $21.41 \pm 0.15 \mathrm{a}, \mathrm{B}, \beta \gamma$ & $39.2 \pm 2.63 \mathrm{a}, \mathrm{A}, \beta$ & $43.09 \pm 1.15 \mathrm{ab}, \mathrm{B}, \gamma$ & $73.65 \pm 3.21 \mathrm{ab}, \mathrm{A}, \gamma$ & $9.85 \pm 0.21 \mathrm{~b}, \mathrm{~A}, \gamma$ & $187.21 \pm 3.51$ \\
\hline & \multirow{3}{*}{ Fillet, Stewed } & $75^{\circ} \mathrm{C}$ & $23.7 \pm 2.2^{\mathrm{a}, \mathrm{A}, \alpha \beta}$ & $32.6 \pm 1.57 \mathrm{~b}, \mathrm{~A}, \beta$ & $39.89 \pm 4.15 \mathrm{a}, \mathrm{A}, \alpha$ & $53.5 \pm 6.48 \mathrm{~b}, \mathrm{~A}, \alpha$ & $7.73 \pm 0.61 \mathrm{a}, \mathrm{A}, \alpha$ & $157.43 \pm 12.06$ \\
\hline & & $85^{\circ} \mathrm{C}$ & $21.59 \pm 1.23 \mathrm{~b}, \mathrm{~A}, \beta \gamma$ & $35.92 \pm 2.33^{b, A}, \alpha \beta$ & $41.39 \pm 2.35 \mathrm{~b}, \mathrm{~B}, \beta, \gamma$ & $66.87 \pm 7.56 \mathrm{~b}, \mathrm{~A}, \mathrm{~B}$ & $8.46 \pm 0.13 \mathrm{~b}, \mathrm{~A}, \mathrm{~B} \gamma$ & $174.23 \pm 8.87$ \\
\hline & & $95^{\circ} \mathrm{C}$ & $20.77 \pm 0.26^{\mathrm{a}, \mathrm{A}, \alpha \beta \gamma}$ & $42.87 \pm 2.30 \mathrm{a}, \mathrm{B}, \beta$ & $42.89 \pm 2.46 \mathrm{a}, \mathrm{B}, \alpha \beta$ & $71.61 \pm 3.50 \mathrm{ab}, \mathrm{AB}, \gamma$ & $9.59 \pm 0.22 \mathrm{~b}, \mathrm{~A}, \gamma$ & $187.73 \pm 2.95$ \\
\hline \multirow{8}{*}{ Norway } & Control(H/G) & & $6.46 \pm 0.6$ & $4.17 \pm 0.09$ & $28.08 \pm 3.23$ & $6.49 \pm 0.05$ & $5.61 \pm 0.5$ & $50.80 \pm 3.49$ \\
\hline & Control(fillet) & & $9.85 \pm 0.81$ & $5.66 \pm 0.41$ & $23.74 \pm 0.26$ & $8.82 \pm 0.07$ & $6.77 \pm 0.28$ & $54.83 \pm 0.94$ \\
\hline & \multirow{3}{*}{$\begin{array}{l}\text { Headed and gutted, } \\
\text { Stewed }\end{array}$} & $75^{\circ} \mathrm{C}$ & $18.06 \pm 1.12 \mathrm{a}, \mathrm{AB}, \alpha \beta$ & $29.97 \pm 0.99 \mathrm{a}, \mathrm{A}, \mathrm{\beta}$ & $39.91 \pm 3.51 \mathrm{a}, \mathrm{A}, \mathrm{B}$ & $43.72 \pm 2.67 \mathrm{~b}, \mathrm{~B}, \alpha$ & $5.86 \pm 0.06 \mathrm{a}, \mathrm{A}, \alpha$ & $137.52 \pm 4.34$ \\
\hline & & $85^{\circ} \mathrm{C}$ & $16.89 \pm 0.91 \mathrm{a}, \mathrm{A}, \alpha$ & $28.88 \pm 1.57 \mathrm{a}, \mathrm{A}, \alpha \beta$ & $41.95 \pm 4.12 \mathrm{~b}, \mathrm{~B}, \beta$ & $48.39 \pm 3.03 \mathrm{a}, \mathrm{A}, \alpha \beta$ & $6.06 \pm 0.55^{a}, \mathrm{~A}, \alpha \beta$ & $142.17 \pm 2.26$ \\
\hline & & $95^{\circ} \mathrm{C}$ & $22.69 \pm 1.25^{b, A}, \gamma$ & $37.77 \pm 1.68 \mathrm{~b}, \mathrm{~B}, \beta$ & $40.03 \pm 0.52 \mathrm{a}, \mathrm{B}, \alpha$ & $76.95 \pm 6.69 \mathrm{a}, \mathrm{AB}, \gamma$ & $6.6 \pm 0.15^{b, A, \gamma}$ & $184.04 \pm 3.33$ \\
\hline & \multirow{3}{*}{ Fillet, Stewed } & $75^{\circ} \mathrm{C}$ & $18.03 \pm 0.65 \mathrm{a}, \mathrm{A}, \alpha \beta$ & $28.21 \pm 2.70 \mathrm{a}, \mathrm{A}, \alpha$ & $35.84 \pm 2.17 \mathrm{~b}, \mathrm{~A}, \gamma$ & $39.11 \pm 0.84^{\mathrm{a}, \mathrm{A}, \alpha}$ & $5.65 \pm 0.27 \mathrm{a}, \mathrm{AB}, \alpha \beta$ & $126.84 \pm 3.06$ \\
\hline & & $85^{\circ} \mathrm{C}$ & $17.11 \pm 0.71 \mathrm{a}, \mathrm{AB}, \alpha$ & $29.24 \pm 1.67 \mathrm{a}, \mathrm{A}, \boldsymbol{\beta}$ & $42.41 \pm 3.58^{b, A}, \beta$ & $45.31 \pm 1.72 \mathrm{a}, \mathrm{A}, \alpha \beta$ & $6.13 \pm 0.51 \mathrm{a}, \mathrm{A}, \alpha$ & $140.2 \pm 1.29$ \\
\hline & & $95^{\circ} \mathrm{C}$ & $22.86 \pm 0.66 \mathrm{~b}, \mathrm{~A}, \gamma$ & $38.53 \pm 0.74 \mathrm{~b}, \mathrm{~A}, \mathrm{~B} \gamma$ & $40.3 \pm 0.45 \mathrm{a}, \mathrm{A}, \mathrm{B}$ & $84.2 \pm 2.53 \mathrm{a}, \mathrm{A}, \gamma$ & $6.64 \pm 0.04 \mathrm{a}, \mathrm{A}, \alpha \beta$ & $192.53 \pm 2.90$ \\
\hline
\end{tabular}

Mean Values with three types of superscripts: ${ }^{a, b}$ _indicate significant differences in origin; ${ }^{A}, \mathrm{~B}$-indicate significant differences in pre-treatment; ${ }^{\alpha, \beta, \gamma}$ _indicate significant differences in Temperature. The significant differences according to Duncan's test $(p<0.05)$.

In the 40 mackerel samples, the concentration ranges of PUT, HIS, TRP, PHE, and SPD were 11.43-43.11, 6.34-84.2, 4.12-45.51, 4.01-42.87, and 2.13-10.09 $\mu \mathrm{g} / \mathrm{g}$, respectively. Among the cooking methods, the level of BAs was highest in stewed mackerel samples $(n=12)$ with $126.84-192.53 \mu \mathrm{g} / \mathrm{g}$. Roasted mackerel had 34.6-93.32 $\mu \mathrm{g} / \mathrm{g}(n=12)$, and fried mackerel $(n=12)$ recorded 33.09-100.28 $\mu \mathrm{g} / \mathrm{g}$. By origin, the total BAs content was higher in pre-treated mackerel from Norway $(51.81 \pm 1.80 \mu \mathrm{g} / \mathrm{g})$ than Korea $(45.09 \pm 1.81 \mu \mathrm{g} / \mathrm{g})$. The BA levels of fillet and H/G fish from Korea were lower than Norway by $14 \%$ and $15 \%$, respectively.

In pre-treated mackerel, the content of total BAs was higher in the fillet (51.01 $\pm 0.94 \mu \mathrm{g} / \mathrm{g})$ than the $\mathrm{H} / \mathrm{G}$ fish $(46.9 \pm 2.38 \mu \mathrm{g} / \mathrm{g})$. In the previous study dealing with biogenic amines formation and its relation to microbiological and sensory attributes in ice-stored whole, gutted, and filleted Mediterranean Sea bass, the number of microorganisms in fillet was more than headed and gutted part [21]. BAs are formed through bacterial enzyme activity. Fresh fish have low levels of BAs, with BA accumulation related to spoilage. The type and level of BAs in fish depend on the extent of spoilage, the specific spoilage organisms, and their counts. The increase in microbial counts due to post-processing contamination and storage time has led to a substantial rise in certain BAs [21].

The reason that stewed mackerel recorded the highest level of BAs $(163.05 \mu \mathrm{g} / \mathrm{g})$ by the cooking method can be explained by the presence of BAs in the soy sauce or soybean paste $[22,23]$. According to Yoon et al., the concentration of BAs was increased in Korean fermented foods [23]. In fermented products, such as soy sauce, soybean paste, and cheese, BAs are formed by the fermentation or decay of foods with a high protein content [19].

In all cooking methods, the level of total BAs increased significantly as the temperature increased. Increases of up to $190 \%, 236 \%$, and $152 \%$ were observed as roasting, frying and stewing temperature increased, respectively ( $p<0.05$ for all). Since there has not been reports about the effect of cooking on the BA formation in fishery products it was hard to evaluate our results. However, in the roasting of cocoa beans, the highest temperature, and air humidity led to the greatest rise in BA due to precursor transformation at high heat treatment [13]. Referring to the study, it is speculated that steamed mackerel had the highest BA content because it had the highest humidity of the three cooking methods. In the study of soybean paste, roasting is attributed to increasing BA [8].

Additionally, recent studies have shown that BA can be formed during heat treatment by chemical decarboxylation of amino acids in the presence of lipid peroxides [24]. Therefore, the frying method seems to have a higher BA content than the roasting method due to the lipid peroxides.

In this study, five BAs, including TRP, PHE, PUT, HIS, and SPD, formed in mackerel subjected to various cooking methods and conditions were analysed. In 40 mackerel samples, the detected concentration ranges of PUT, HIS, TRP, PHE, and SPD were 11.43-43.11, 
6.34-84.2, 4.12-45.51, 4.01-42.87, and 2.13-10.09 $\mu \mathrm{g} / \mathrm{g}$, respectively. The total BAs formed during roasting, frying, and stewing were 42.01-93.32, 33.09-146.58, and 126.84-19.53 $\mu \mathrm{g} / \mathrm{g}$, respectively. The total level of BAs increased significantly with increasing temperature in all cooking methods. Compared to the control group, the total BAs for roasting, frying, and stewing increased up to $170 \%, 311 \%$, and $398 \%$, respectively, when cooking was completed.

The results of this study indicate that a change of the cooking conditions such as temperature and time may contribute to the reduction of BA concentration in foods. Future studies should include a comprehensive analysis of the BA levels in foods using various western and eastern cooking recipes for controlling the formation and dietary exposure to BAs.

Author Contributions: Conceptualization, K.-G.L. and Y.-S.K.; methodology, Y.-S.K. and H.P.; software, H.P.; validation, Y.K. and H.P.; formal analysis, Y.-S.K. and J.P.; investigation, Y.-S.K.; resources, Y.K.; data curation, Y.-S.K.; writing—original draft preparation, Y.-S.K.; writing—review and editing, Y.-S.K.; visualization, J.P.; supervision, K.-G.L.; project administration, K.-G.L.; funding acquisition, K.-G.L. All authors have read and agreed to the published version of the manuscript.

Funding: This work was supported by the the Basic Science Research Program through the National Research Foundation of Korea [grant number NRF, 2021R1A2B5B01002296] and Korea Institute of Planning and Evaluation for Technology in Food, Agriculture, Forestry and Fisheries [IPET, No. 320110-01].

Institutional Review Board Statement: Not applicable.

Informed Consent Statement: Not applicable.

Acknowledgments: The authors would like to thank the support of Dongguk University.

Conflicts of Interest: The authors declare that there is no conflict of interest.

\section{References}

1. Kim, B. An analysis of the consumer perception on imported fisheries products after implementation of FTAs: Focusing on main products. J. Fishries Mar. Sci. Educ. 2018, 30, 633-642. [CrossRef]

2. Kim, Y.; Kim, B.-H.; Kim, K.-A.; Kim, D.-H.; Yun, H.-J.; Kwak, S.-H.; Kang, K.-J.; Cho, W.-H.; Moh, A.-R.; Choi, O.-K.; et al. Safety assessment of biogenic amines in school-meal fishery products. J. Food Hyg. Saf. 2020, 35. [CrossRef]

3. Bilgin, B.; Gençcelep, H. Determination of biogenic amines in fish products. Food Sci. Biotechnol. 2015, 24, 1907-1913. [CrossRef]

4. Preti, R.; Rapa, M.; Vinci, G. Effect of steaming and boiling on the antioxidant properties and biogenic amines content in green bean (Phaseolus vulgaris) varieties of different colours. J. Food Qual. 2017, 2017, 1-8. [CrossRef]

5. Doeun, D.; Davaatseren, M.; Chung, M.-S. Biogenic amines in foods. Food Sci. Biotechnol. 2017, 26, 1463-1474. [CrossRef]

6. Moon, J.S.; Kim, Y.; Jang, K.I.; Cho, K.-J.; Yang, S.-J.; Yoon, G.-M.; Kim, S.-Y.; Han, N.S. Analysis of biogenic amines in fermented fish products consumed in Korea. Food Sci. Biotechnol. 2010, 19, 1689-1692. [CrossRef]

7. Park, J.S.; Lee, C.H.; Kwon, E.Y.; Lee, H.J.; Kim, J.Y.; Kim, S.H. Monitoring the contents of biogenic amines in fish and fish products consumed in Korea. Food Control 2010, 21, 1219-1226. [CrossRef]

8. Yoon, S.; Kim, M.; Moon, B. Various biogenic amines in Doenjang and changes in concentration depending on boiling and roasting. Appl. Biol. Chem. 2017, 60, 273-279. [CrossRef]

9. Shalaby, A.R. Significance of biogenic amines to food safety and human health. Food Res. Int. 1996, 29, 675-690. [CrossRef]

10. Yeh, C.-Y.; Lin, S.-J.; Hwang, D.-F. Biogenic amines and histamine of marlin fillet and spotted mackerel fillet sampled from cafeteria and anchovy from fish market in Keelung. J. Food Drug Anal. 2004, 12, 128-132.

11. Oliveira, S.D.; Franca, A.S.; Glória, M.B.A.; Borges, M.L.A. The effect of roasting on the presence of bioactive amines in coffees of different qualities. Food Chem. 2005, 90, 287-291. [CrossRef]

12. Ishibashi, Y.; Hanyu, N.; Nakada, K.; Suzuki, Y.; Yamamoto, T.; Takahashi, T.; Kawasaki, N.; Kawakami, M.; Matsushima, M.; Urashima, M. Endothelin protein expression as a significant prognostic factor in oesophageal squamous cell carcinoma. Eur. J. Cancer 2003, 39, 1409-1415. [CrossRef]

13. Oracz, J.; Nebesny, E. Influence of roasting conditions on the biogenic amine content in cocoa beans of different Theobroma cacao cultivars. Food Res. Int. 2014, 55, 1-10. [CrossRef]

14. Visciano, P.; Schirone, M.; Tofalo, R.; Suzzi, G. Biogenic amines in raw and processed seafood. Front. Microbiol. 2012, 3, 188. [CrossRef] [PubMed]

15. Biji, K.; Ravishankar, C.; Venkateswarlu, R.; Mohan, C.; Gopal, T.S. Biogenic amines in seafood: A review. J. Food Sci. Technol. 2016, 53, 2210-2218. [CrossRef] 
16. Prester, L. Biogenic amines in fish, fish products and shellfish: A review. Food Addit. Contam. Part A 2011, 28, 1547-1560. [CrossRef] [PubMed]

17. Naila, A.; Flint, S.; Fletcher, G.; Bremer, P.; Meerdink, G.J.J.O.F.S. Control of biogenic amines in food-Existing and emerging approaches. J. Food Sci. 2010, 75, R139-R150. [CrossRef]

18. Chong, C.Y.; Bakar, F.A.; Rahman, R.A.; Bakar, J.; Zaman, M.Z. Biogenic amines, amino acids and microflora changes in Indian mackerel (Rastrellinger kanagurta) stored at ambient $\left(25-29^{\circ} \mathrm{C}\right)$ and ice temperature $\left(0^{\circ} \mathrm{C}\right)$. J. Food Sci. Technol. 2014, 51, 1118-1125. [CrossRef]

19. Lee, J.-Y.; Kim, Y.-g.; Her, J.-Y.; Kim, M.K.; Lee, K.-G. Reduction of biogenic amine contents in fermented soybean paste using food additives. LWT 2018, 98, 470-476. [CrossRef]

20. Ben-Gigirey, B.; De Sousa, J.M.V.B.; Villa, T.G.; Barros-Velazquez, J. Changes in biogenic amines and microbiological analysis in albacore (Thunnus alalunga) muscle during frozen storage. J. Food Prot. 1998, 61, 608-615. [CrossRef]

21. Paleologos, E.K.; Savvaidis, I.N.; Kontominas, M.G. Biogenic amines formation and its relation to microbiological and sensory attributes in ice-stored whole, gutted and filleted Mediterranean Sea bass (Dicentrarchus labrax). Food Microbiol. 2004, 21, 549-557. [CrossRef]

22. Han, G.-H.; Bahn, K.-N.; Son, Y.-W.; Jang, M.-R.; Lee, C.-H.; Kim, S.-H.; Kim, D.-B.; Kim, S.-B.; Cho, T.-Y. Evaluation of biogenic amines in Korean commercial fermented foods. Korean J. Food Sci. Technol. 2006, 38, 730-737.

23. Yoon, H.; Park, J.H.; Choi, A.; Hwang, H.-J.; Mah, J.-H. Validation of an HPLC analytical method for determination of biogenic amines in agricultural products and monitoring of biogenic amines in Korean fermented agricultural products. Toxicol. Res. 2015, 31, 299-305. [CrossRef] [PubMed]

24. Zamora, R.; Delgado, R.M.; Hidalgo, F.J. Formation of $\beta$-phenylethylamine as a consequence of lipid oxidation. Food Res. Int. 2012, 46, 321-325. [CrossRef] 